\title{
Modeling and Characterization of Leakage Inductances for Transformer Winding Fault Studies
}

\author{
Luís M.R. Oliveira ${ }^{1,3}$ and A.J. Marques Cardoso ${ }^{2,3}$ \\ ${ }^{1}$ Instituto Superior de Engenharia, Universidade do Algarve, Faro, Portugal \\ ${ }^{2}$ University of Beira Interior, Department of Electromechanical Engineering, Portugal \\ ${ }^{3}$ Instituto de Telecomunicações, Department of Electrical and Computer Engineering, \\ University of Coimbra, Pole II, P - 3030-290 Coimbra, Portugal \\ lolivei@ualg.pt, ajmcardoso@ieee.org
}

\begin{abstract}
This paper presents an analytical method to compute the leakage inductances of power transformers with a turn-to-turn winding fault. A leakage inductance model to represent the transformer with faulty turns is also proposed. The results obtained from the application of the analytical method are validated by using data obtained from finite-element analysis and experimental tests.
\end{abstract}

Keywords: Transformers, winding faults, leakage inductances, modeling.

\section{Introduction}

Power transformers are key elements of the electric generation, transmission and distribution network and constitute one of the most capital-intensive investments made by power system utilities. The unexpected failure of a power transformer can generate substantial costs for repair and financial loss due to unscheduled electrical outage. Therefore, it is of crucial importance to detect internal defects in their incipient stage, so that the faulted unit can be immediately disconnected, avoiding the progression of the defective condition into a catastrophic failure, minimizing the damages in the transformer and other expensive neighboring equipment, and thus reducing downtime and total outage costs.

The development of new techniques for transformer condition monitoring and fault protection requires a detailed characterization of the transformer behavior during the occurrence of turn-to-turn winding short-circuits. The experimental study of these incipient internal faults presents some difficulties, mainly due to the high magnitudes of the faulty currents involved, which can damage the test transformer. Therefore, a detailed analysis of these phenomena can be better investigated by the use of a suitable digital simulation transformer model [1].

Several circuit-based transformer models were presented in the last few years for winding fault studies [1]-[4]. One of the major difficulties in these approaches is to model the leakage inductances when the turn-to-turn short-circuit is present. The difficulty arises because the distribution of the magnetic flux is substantially modified 
when such a fault occurs [5]. In [2] the method to determine the leakage inductances relies on correction factors, in order to take into account the radial component of the leakage flux. In [3] an analytical formula for the leakage inductance of the faulty winding is developed, but only for the simpler case of transformer no-load conditions. Nevertheless, the derivation of the models is not straightforward and difficult to be implemented. More recently a simplified method was proposed [4], in which the leakage inductances are computed from the nameplate short-circuit reactance. Errors up to $68 \%$ are reported (as compared with experimental results) and only a model based on finite elements method would yield good results.

This work presents an analytical method to compute the leakage inductances of power transformers with a turn-to-turn winding fault. The influence of the fault position and fault progression is also characterized. Additionally, a leakage inductance equivalent circuit to represent the transformer with faulty turns is proposed. The results obtained from the application of the analytical method are validated by using data obtained from finite element analysis and experimental tests.

\section{Relationship to Internet of Things}

The smart grid can be seen as one of the Internet of Things application domains, in which intelligent electronic devices and their communication capabilities can be used to provide unprecedented reliability levels in the power network. However, for this to occur it is necessary the development of new fault detection methods, which must be integrated with the smart grid technologies. This in turn requires detailed and extensive modeling and simulation of the power system network. A power transformer model for winding fault studies is essential for these purposes.

\section{$3 \quad$ Winding Fault Characterization}

For the experimental investigation a three-phase, two winding, three limb transformer, of $10.3 \mathrm{kVA}, 230 / 132 \mathrm{~V}$, was used. The primary and the secondary windings have 152 and 90 turns, respectively. In each transformer winding there are five additional tappings connected to the coils, allowing for the introduction of shorted turns at several locations in the winding, as shown in Fig. 1(a), for one phase of the primary and the secondary windings. Only the coils of the center limb were used to perform the short-circuit single-phase tests, which results in a shell-type core design.

When a fault occurs in the primary-side, the short-circuited turns act as an autotransformer load on the winding, as shown in Fig. 1(b). The initial effect of the inter-turn short circuit is limited to a slight increase in the primary current. However, the insulation failure can lead to a high circulating current in the shorted turns, even if only a small number of turns is affected.

Fig. 2(a) presents the current waveforms in the transformer windings for the case of a load-test during the occurrence of a turn-to-turn fault in the primary winding. To protect the transformer from complete failure when the fault was introduced the current in the shorted turns is limited by an auxiliary resistor, which represents the 
fault contact resistance, $R_{s h}$. It can be seen that the current in the shorted turns $\left(i_{b}\right)$ and the current in the secondary winding $\left(i_{s}\right)$ are in phase opposition with the primary-side current (i.e., they are opposing the primary magnetomotive force (MMF)).

The leakage inductance is usually obtained by performing the short-circuit test. It might be thought that the effect of the inter-turn short-circuit could be analyzed by performing a short-circuit test where both $N_{s}$ and $N_{b}$ turns were individually shorted, Fig. 1(c). However, as explained next, this test is far from representing the true leakage flux distribution. The resultant current waveforms obtained for this case are shown in Fig. 2(b). It can be seen that the current $i_{b}$ presents a completely different behavior than the one for the load test: it has a very small magnitude and is opposing the secondary MMF, instead of opposing the primary MMF. The main reason is that the mutual flux that links the three coils $\left(N_{a}, N_{b}\right.$ and $\left.N_{s}\right)$ is significantly different in these two tests, and, therefore, the induced currents do not follow the same pattern. As a result the flux distribution obtained by this short-circuit test is very different from the leakage flux component in the load test and it is not representative of this latter condition.

A better solution to study these phenomena can be obtained by performing the short-circuit test of Fig. 1(d), where the short is applied to $N_{s}$ and $N_{b}$ connected in series-addition. With this approach both currents in these coils are opposing the primary magnetomotive force, Fig. 2(c), which is much more similar to the results of the load-test of Fig. 2(a).

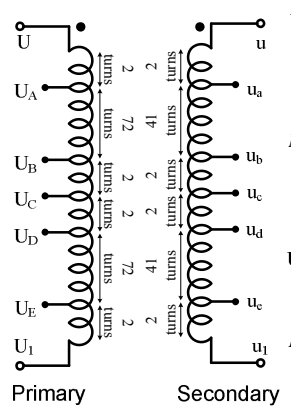

(a)

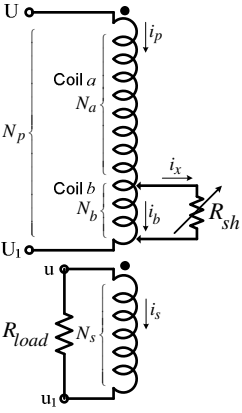

(b)

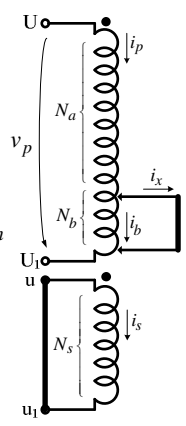

(c)

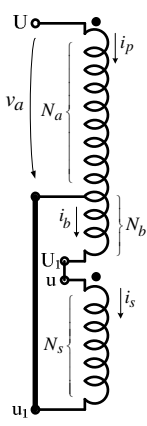

(d)

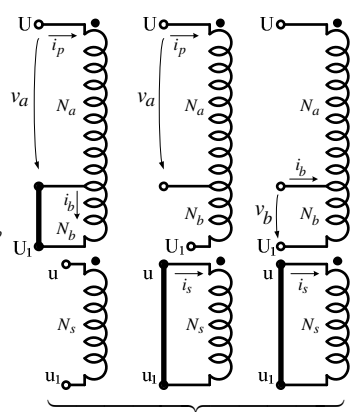

(e)

Fig. 1. (a) Location of the tappings of the windings; (b) equivalent circuit for a fault occurring in the primary winding; (c), (d) and (e) short-circuit test schematics
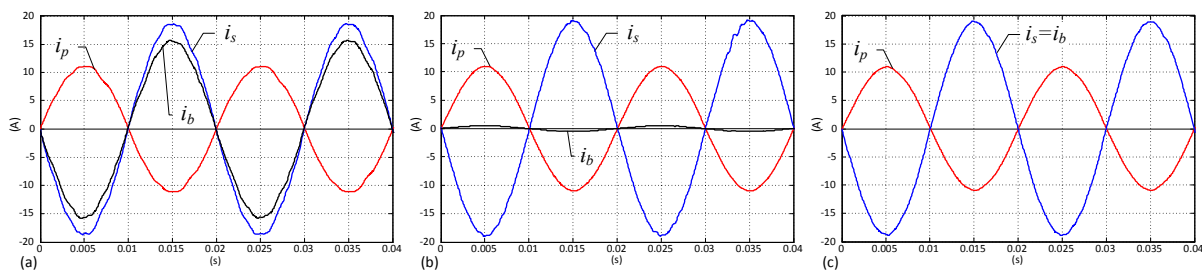

Fig. 2. Current waveforms for the case of: (a) load-test of Fig.1(b); (b) short-circuit test of Fig. 1(c); (c) short-circuit test of Fig. 1(d). ( $N_{b}=2$ turns.) 
Alternatively, the leakage inductances can be obtained by performing the traditional short-circuit test were one winding is shorted at a time, Fig. 1(e). With this approach the three-winding transformer theory can be applied to obtain the leakage inductance transformer model, which must be consistent with the conditions of the non-standard short-circuit test of Fig. 1(d).

\section{$4 \quad$ Leakage Inductance Analytical Computation}

Several formulas for the analytical computation of the leakage inductances have been proposed in the past. For the case of concentric transformer windings, with the same height $h$, the leakage flux that flows due to the load current is virtually parallel to the axis (except in the ends of the windings). Under this assumption, Fig. 3(a) shows the per unit MMF distribution $\left(m_{k}\right)$ obtained for the windings geometric structure of the test transformer. It is considered that the MMF's of the two windings are equal and opposite, which is valid for normal operating conditions (no fault). The leakage inductance can be computed by using (notation as per Fig. 3) [6]:

$$
L_{\sigma(a x)}=K_{\sigma} \frac{2 \pi N_{1}^{2} \mu_{0}}{h} \sum_{k=1}^{n}\left[m_{k}^{2} \cdot g_{k} \cdot r_{g k}+\left(m_{k}^{2}+m_{k-1}^{2}+m_{k} \cdot m_{k-1}\right) \cdot w_{k} \cdot r_{w k} / 3\right]
$$

where $N_{1}$ is the number of turns of the excited winding, $\mu_{0}$ is the permeability of free space, $n$ is the number of vertical layers ( 7 in this case), $r_{w k}$ is the mean radio of the coil $k, r_{g k}$ is the mean radio of the gap between coils $k$ and $k+1$, and $m_{k}$ is the per unit MMF acting on gap between coils $k$ and $k+1$, see Fig. 3(a).The Rogowski correction factor, $K_{\sigma}$, is used to take into account the flux fringing at the top and bottom of the windings and the effect of the iron core. For the case of a shell-type design:

$$
K_{\sigma}=1-\frac{1-e^{-\frac{2 \pi E}{T}}}{2 \pi E / T}\left[1-\frac{1-e^{-\frac{2 \pi E}{T}}}{2 e^{\frac{4 \pi C}{T}}}\left[\frac{L_{2}}{L}+\frac{L_{1}}{L}\left(1+e^{-\frac{2 \pi\left(C_{1}-C\right)}{T}}\right)\right]-\frac{L_{1}}{L} e^{-\frac{2 \pi\left(E+2 C+2 C_{1}\right)}{T}}\right]
$$
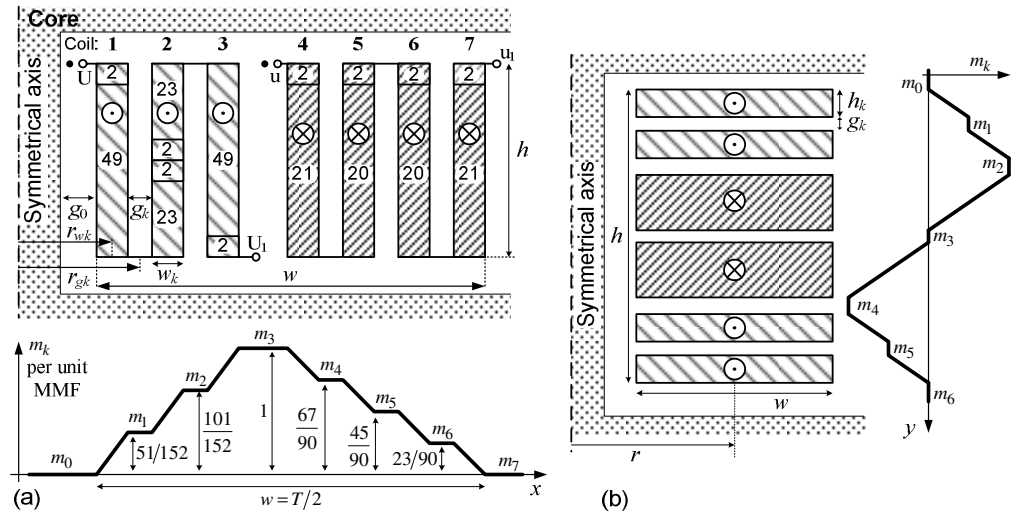

(b)

Fig. 3. Geometric structure and per unit leakage MMF distribution for the case of: (a) axial configuration; (b) radial configuration 
being $E$ the height of the winding, $T$ the wave length of the MMF wave, $L$ the mean coil perimeter, $L_{1}$ that part of the perimeter which has iron on both sides, $L_{2}$ the rest of the coil perimeter, and $C$ and $C_{1}$ the distances from iron to coil on the two sides [6].

Equation (1) can also be used, with proper adaptations, for the computation of the leakage inductance of a disk-type winding configuration. Fig. 3(b) presents a generic disk-type winding arrangement. In this case it can be assumed that the leakage flux has only one component in the radial direction and the radial leakage inductance becomes:

$$
L_{\sigma(\text { rad })}=K_{\sigma} \frac{2 \pi N_{1}^{2} \mu_{0} r}{w} \sum_{k=1}^{\mathrm{n}}\left[m_{k}^{2} \cdot g_{k}+\left(m_{k}^{2}+m_{k-1}^{2}+m_{k} \cdot m_{k-1}\right) \cdot h_{k} / 3\right]
$$

When there are irregularities in the concentric windings, such as a fault, the leakage flux is no longer parallel to the axis, but has significant components of radial flux, depending on the amount of asymmetry [7]. The formulas given by (1) and (3) are no longer valid under these asymmetrical conditions and a direct computation of the leakage inductance for these winding arrangements can be very complicated and extremely laborious. A very ingenious and useful way of dealing with these situations was proposed by Stephens [7] (and generalized in [6]), in which the leakage inductance is divided into two components, one axial and the other radial. Each component can be computed separately and then added together to give, very nearly, the value of the total leakage inductance. Fig. 4 illustrates the basic principle of the method with a simplified diagram, assuming a fault in the middle of the primary winding. The axial component is obtained by dividing the affected winding into two coils, one referring to the healthy portion of the winding and the other to the faulty part. These two parts are uniformly distributed along the axis, resulting in a concentric design configuration, which can be computed by (1).

The procedure to obtain the leakage inductance radial component is shown in Fig. 4(b). First, the secondary-side current is converted to the primary-side. Next, the secondary winding is divided into three segments, with the same dimensions of those in the primary winding. The MMF of each segment is then computed assuming an uniform distribution of the ampere-turns in the windings. Finally, the MMF's of the corresponding segments of each winding are summed up and the radial component of the leakage inductance can be computed by using (3).

The total leakage inductance is then obtained:

$$
L_{\sigma}=L_{\sigma(a x)}+L_{\sigma(\text { rad })}
$$

A finite elements method (FEM) based transformer model [8] is also used to investigate the adequacy of the analytical calculations. The energy method was used to compute the leakage inductance from the FEM results [9].

Fig. 5(a) presents the results obtained when the position of the faulty turns $\left(N_{b}\right)$ are moved along the vertical axis of coil 1, from top to bottom, using the series-addition short-circuit test of Fig. 1(d). The leakage inductance takes greater values when the $N_{b}$ turns are located at the coil ends, because the radial component is higher in this situation. Obviously, the asymmetry increases with the number of the faulty turns, and, as a 
consequence, there is also an increase in the variation of the leakage inductance when the $N_{b}$ turns are moved along the winding. Only one experimental result can be obtained for this specific test conditions, due to the fixed tap positions in the coils.

Fig. 5(b) presents the variation of the leakage inductance when the fault progresses vertically, involving additional turns, first from the top to bottom in coil 1, and then affecting the neighboring vertical layer (coil 2). The leakage inductance initially grows, due the increasing values of the radial component. It is interesting to note that this behavior is opposing the fault progression, since it tends to limit the faulty current. The radial component reaches its highest level when the fault has extended to just about the vertical center of the coil and then decreases more or less symmetrically. This pattern is repeated in the other vertical layers (only shown for coils 1 and 2). The axial component of the leakage inductance drops, since the number of primary turns is effectively decreasing as the fault evolves.

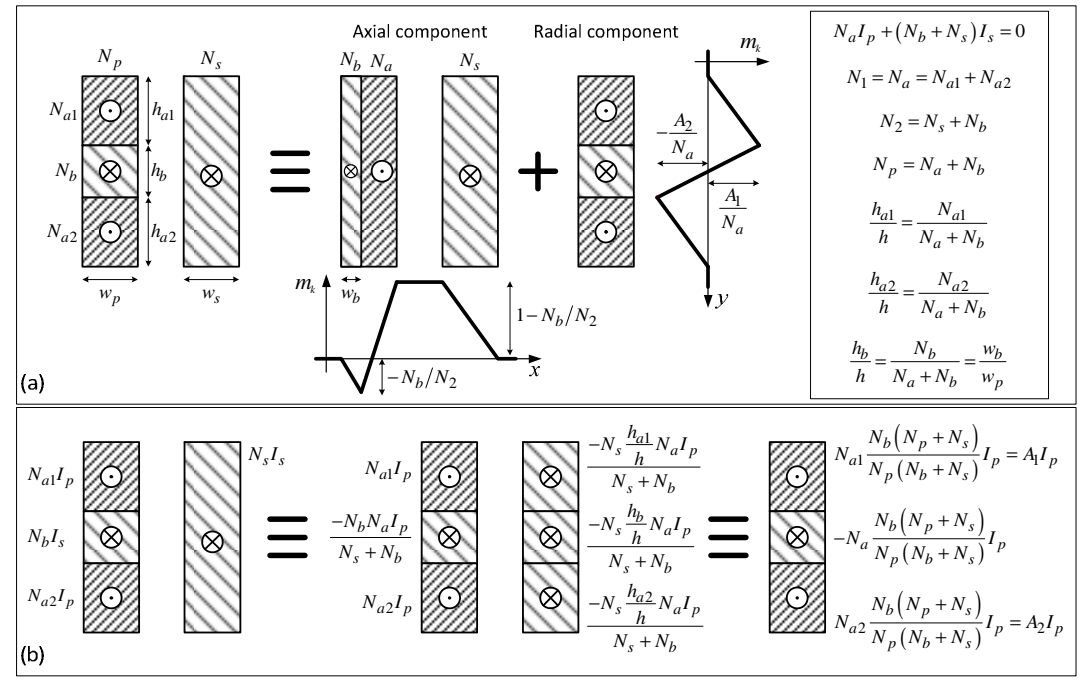

Fig. 4. (a) Equivalent representation of a fault in terms of two components, axial and radial; (b) diagram illustrating the procedure to obtain the radial component

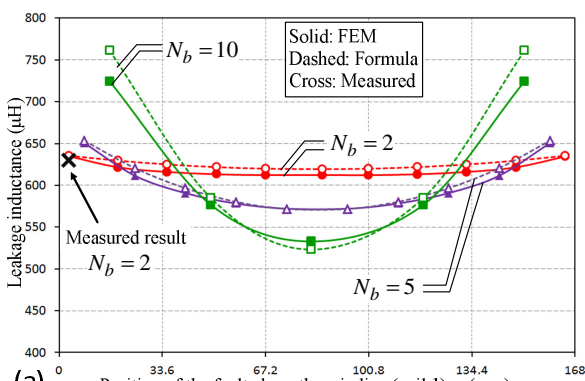

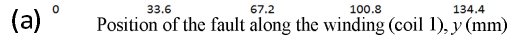

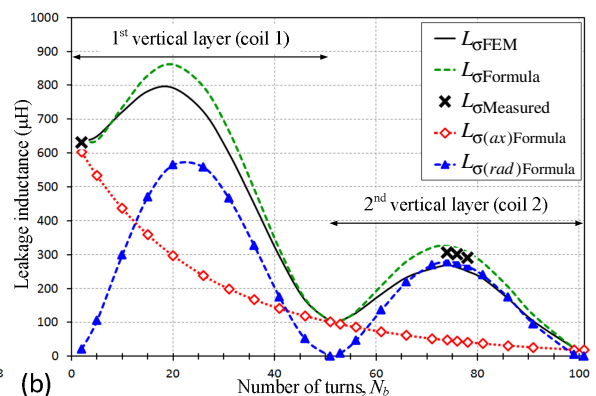

(b) Fig. 5. Leakage inductance as a function of the: (a) number of $N_{b}$ turns and their relative
position along the winding (coil 1); (b) fault progression. (Short-circuit test of Fig. 1(d).) 
The results obtained by the analytical calculation, the FEM analysis, and the experimental tests are in relatively good agreement.

The aforementioned method is also valid for computing the leakage inductances between pairs of windings for the case of the standard short-circuit tests of Fig. 1(e). Fig. 6(a) presents the corresponding results as a function of the $N_{b}$ turns, which are also in good agreement with the FEM analysis and the measured values $\left(L_{\sigma(i j)} \equiv\right.$ leakage inductance when the $N_{i}$ turns are excited and the $N_{j}$ turns are shorted).

\section{Leakage Inductance Equivalent Circuit Model}

The equivalent circuit for the leakage inductance of three-winding transformers proposed in [10] is adapted here to represent the transformer with faulty turns. The equivalent circuit is shown in Fig. 7 and its parameters are computed from the short-circuit inductances between the pairs of windings obtained by the tests of Fig. 1(e):

$$
\begin{gathered}
L_{\sigma 1}=L_{\sigma(a b)} \\
L_{\sigma 2}=L_{\sigma(b s)}\left(N_{a} / N_{b}\right)^{2} \\
M_{\sigma}=\left[L_{\sigma(a s)}-L_{\sigma(a b)}-L_{\sigma(b s)}\left(N_{a} / N_{b}\right)^{2}\right] / 2 .
\end{gathered}
$$

The leakage inductance equivalent circuit model can be used to simulate the short-circuit test of Fig. 1(d). By analyzing the circuit of Fig. 7, with a short applied to the seriesconnected $N_{b}$ and $N_{s}$ turns, the equivalent inductance becomes:

$$
L_{\sigma(\mathrm{a}, \mathrm{b}+\mathrm{s})}=L_{\sigma 1}+L_{\sigma 2}\left[N_{s} /\left(N_{s}+N_{b}\right)\right]^{2}+2 M_{\sigma} N_{s} /\left(N_{s}+N_{b}\right)
$$

Fig. 6(b) compares the results obtained by applying (8) and the ones previously presented in Fig. 5(b). Globally, the proposed leakage inductance model yields good and consistent results. The FEM results are almost coincident. Minor differences can be detected in the analytical computed results, mainly due to approximations in the calculation of the mean radius and the Rogowski correction factors.

The leakage inductance network of Fig. 7 can be integrated with other transformer models [1], [10], in order to take into account the core and loss components.
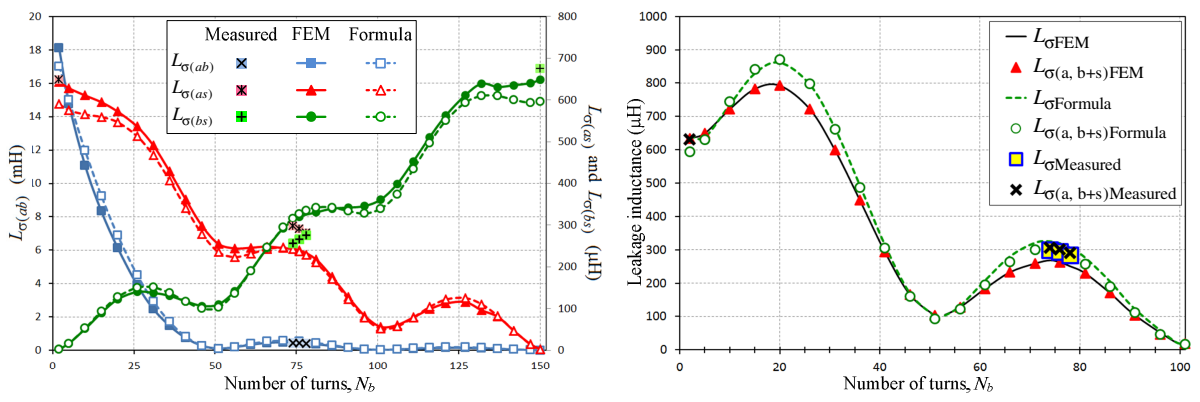

Fig. 6. (a) Leakage inductance as a function of the number of $N_{b}$ turns for the case of the shortcircuit tests of: (a) Fig. 1(e); (b) Fig. 1(d) 


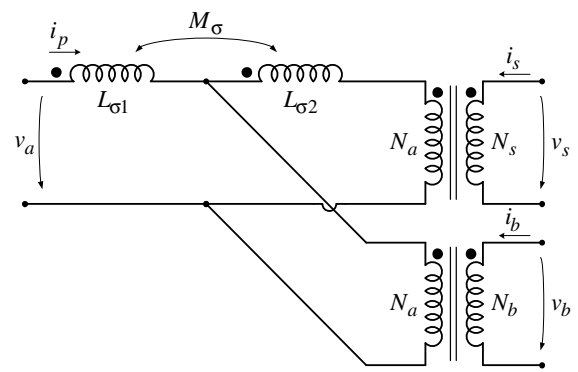

Fig. 7. Leakage inductance equivalent circuit

\section{Conclusions}

This paper has presented a method for the analytical determination of the leakage inductances of transformers with winding interturn short-circuits. An equivalent circuit for the representation of the leakage inductance of transformers with winding faults is also proposed. The experimental and FEM analysis results confirm the adequacy of the proposed analytical calculation method.

Work is currently in progress to further simplify the leakage inductances computation method, in order to allow their determination from nameplate data and core window dimensions.

\section{References}

1. Oliveira, L.M.R., Cardoso, A.J.M.: A Permeance-Based Transformer Model and its Application to Winding Interturn Arcing Fault Studies. IEEE Trans. Power Delivery 25, 1589-1598 (2010)

2. Bastard, P., Bertrand, P., Meunier, M.: A Transformer Model for Winding Fault Studies. IEEE Trans. Power Delivery 9, 690-699 (1994)

3. Jablonski, M., Napieralska-Juszczak, E.: Internal Faults in Power Transformers. IET Electric Power Applications 1, 105-111 (2007)

4. Avendaño, A., Mork, B.A., Høidalen, H.K.: Transformer Internal Fault Modeling in ATP. In: Int. Conf. Power Systems Transients (2011)

5. Billig, E.: Mechanical Stresses in Transformer Windings. Journal IEE, Part II 93, 227-243 (1946)

6. Blume, L.F. (ed.): Transformer Engineering, 2nd edn. John Wiley \& Sons (1951)

7. Stephens, H.O.: Transformer Reactance and Losses with Nonuniform Windings. AIEE Trans. 53, 346-349 (1934)

8. Meeker, D.C.: Finite Element Method Magnetics, Version 4.2, User's Manual (2010)

9. Kulkarni, S.V., Khaparde, S.A.: Transformer Engineering: Design and Practice. Marcel Dekker (2004)

10. León, F., Martinez, J.A.: Dual Three-Winding Transformer Equivalent Circuit Matching Leakage Measurements. IEEE Trans. Power Delivery 24, 160-168 (2009) 